\title{
Infant and Young Child Feeding Practice in Nepal
}

\author{
Anil Kumar Sah
}

BPH 22nd Batch

Infant and young child feeding is critical for child health and survival. WHO and UNICEF recommend that infants be exclusively breastfed for the first 6 months of life and thereafter receive adequate complementary foods in addition to continued breastfeeding until 2 years of age or beyond. Appropriate complementary feeding is critical for the achievement of healthy growth and development and mere survival of young children.

Under nutrition is responsible for at least $35 \%$ of under-5 deaths. It is also a direct cause of mortality, and a major disabler preventing children who survive to reach their full developmental potential. An estimated $32 \%$ of children less than 5 years of age in developing countries are stunted and $10 \%$ are wasted. Lack of appropriate breastfeeding and complementary feeding practices are main causes of under nutrition. Inappropriate breastfeeding and complementary feeding practices can also initiate the problem of overweight and obesity that may only become most apparent in children beyond the age of 2 years. Many countries suffer from the double burden of both types of malnutrition.

High rates of exclusive breastfeeding during the first 6 months of life and continued breastfeeding with complementary feeding can potentially prevent $13 \%$ and $6 \%$ respectively of under-5 deaths each year. Recently, increasing emphasis has been placed on exclusive breastfeeding for infants up to 6 months of age as an important factor for child survival, growth and development. However, continued breastfeeding is also very critical to improve feeding in children 6-23 months of age, as breast milk is an important source of energy and nutrients in the child's diet and has numerous other beneficial effects.

IYCF policy has been just approved by the Government National Plan of Action. The policy mentions no plan of action and it is not funded adequately. It is recommended that there should be a national plan of action of the policy and there should be adequate fund for it. $6.9 \%$ of hospitals and maternity facilities that provide maternity services have been designated "Baby Friendly" for implementing ten steps to successful breastfeeding.

There has been no activity in achieving baby friendly targets for last 10 years and hence the dismal number of hospital certified as Baby Friendly. There has been no regular followup assessment as well. Not enough health workers have been trained in breastfeeding. It is therefore high time that the training of health workers conducted as soon as practicable there by increasing the number of Baby Friendly Hospitals.

The code of marketing of breast milk substitute though in place has not been in effect. Only some articles of code are in place as law. The code of marketing should be strictly monitored and committee to look after such activity should be formed. The curricula of health workers do not adequately cover infant and young infant feeding. There is to some degree mother friendly child birth procedures and are trying to reach all the health facilities. Infant feeding is taught in IMCI package but has recovered 25 of 75 districts of Nepal. There is need to revise the curricula of all the health workers in order to include the infant feeding. In service training should be provided to all level of health providers.

There is to some degree access to counseling on infant feeding during and after pregnancy. There is need to train more counselors on infant feeding and such training package should be integrated into national child health strategy. There is to some degree national IEC materials covering infant and young child feeding. The IMCI program also covers to some extent on this aspect. There should be a national IEC strategy for infant and young child feeding and this should be implemented from community level. To promote IEC strategy electronic and print media should be utilized.

National PMCT guideline has just been implemented in few selected hospitals that include HIV and feeding strategy. Most of the health workers have not received training on HIV and feeding policies. Hence there is need to implement PMCT in all hospital and all health workers should be trained in HIV and feeding policies.

Complementary feeding behaviors vary substantially across caste/ethnic groups and that even with these groups; the pattern of feeding across wealth categories varies. Bahun/ Chhetri is in the poorest and richest group (60\% and $80 \%$ respectively) but fewer $40 \%$ in Dalits and Muslim. It seems likely that ethnicity, higher income, or perhaps better education, in more wealthy groups allows Bahun/Chhetri to provide better diet.

There are no policies that address issues related to infant and young child feeding in emergencies and hence there is need to develop policies regarding the same during the time of emergencies.

\section{Infant and young child nutrition}

The Fifty-fourth World Health Assembly, deeply concerned to improve infant and young child nutrition and to alleviate all forms of malnutrition in the world, because more than one-third of under-five children are still malnourished -whether stunted, wasted, or deficient in 
iodine, vitamin A, iron or other micronutrients - and because malnutrition still contributes to nearly half of the 10.5 million deaths each year among preschool children worldwide.

Deeply alarmed that malnutrition of infants and young children remains one of the most severe global public health problems, a t once a major cause and consequence of poverty, deprivation, food insecurity and social inequality, and that malnutrition is a cause not only of increased vulnerability to infection and other diseases, including growth retardation, but also of intellectual, mental, social and developmental handicap, and of increased risk of disease throughout childhood, adolescence and adult life.

Recognizing the right of everyone to have access to safe and nutritious food, consistent with the right to adequate food and the fundamental right of everyone to be free from hunger, and that every effort should be made with a view to achieving progressively the full realization of this right; Acknowledging the need for all sect ors of society including governments, civil society, health professional associations, nongovernmental organizations, commercial enterprises and international bodies - to contribute $\mathrm{t} o$ improved nutrition for infants and young children by using every possible means at $\mathrm{t}$ heir disposal, especially by fostering optimal feeding practices, incorporating a comprehensive multisectoral, holistic and strategic approach.

Noting the guidance of the Convention on the Rights of the Child, in particular Article 24, which recognizes, interalia, the need for access to and availability of both support and information concerning the use of basic knowledge of chi ld health and nutrition, and the advantages of breast feeding for all segment s of society, in particular parent $s$ and children;

Conscious that despite the fact that the International Code of Marketing of Breast-milk

Substitutes and relevant, subsequent Health Assembly resolutions state that there should be no

Advertising or other forms of promotion of products within its scope, new modern communication methods, including electronic means, are currently increasingly being used to promote such products

Exclusive Breast Feeding, by age in months (DHS)

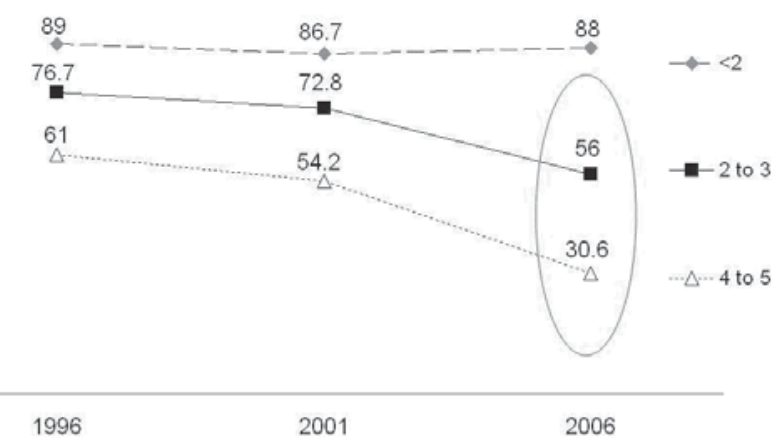

Nepal has done extensive work on promoting exclusive breastfeeding, and much has been achieved. However, as described by NPAN'07, results of DHS surveys in 1996, 2001, and 2006 indicate that exclusive breastfeeding (EBF) of infants to 6 months of age is gradually declining. Reviewing these data by month of age shows that the decrease in EBF appears to have occurred only in infants after two months, i.e. EBF in infants less than 2 months remained at almost 90\% (6) (Figure). Improving EBF rates will require better understanding the reasons for the decrease in older infants, and the government may need to modify their current strategies to address these reasons. Progress with complementary feeding has been slower. The recent DHS suggests that $56 \%$ of families use adequate IYCF practices.

\section{Conclusion}

There was lack of aware about infant and young child feeding practice in Nepal. Good practice is in upper caste than other caste so provide information about feeding practice in all ethnicity. Baby breast feeding initiating programme will improve if breast feeding initiating in baby friendly hospital and also provide counseling about PMTCT for improving better feeding practice. For improving better feeding practice are required minimizing hindering factors of feeding practices.

\section{References}

1. The optimal duration of exclusive breastfeeding. Report of an Expert Consultation. Geneva, World Health Organization, 2001 (http://www.who.int/ child_adolescent_health/documents/nhd_01_09/en/ index.html).

2. WHO/UNICEF. Global strategy on infant and young child feeding. Geneva, World Health Organization, 2003 (http://www.who.int/child_adolescent_health/ documents/9241562218/ en/index.html).

3. $\mathrm{PAHO} / \mathrm{WHO}$. Guiding principles for complementary feeding of the breastfed child. Washington DC,Pan American Health Organization/World Health Organization, 2003 (http://www.who.int/ child_adolescent_health/documents/a85622/en/ index.html).

4. Black R et al. Maternal and child undernutrition: global and regional exposures and health consequences. Lancet, 2008, 371(9608):243-260.

5. Jones $\mathrm{G}$ et al. How many child deaths can we prevent this year? Lancet, 2003; 362:65-71.

6. Steve Hodgins, personal communication, May 2009. 